\title{
Erratum to: Propagation and Evolution of Strain Localization in Clay
}

Dunja Perić and Xingdong Wu

\section{Erratum to:}

Chapter 'Propagation and Evolution of Strain Localization in Clay' in: E. Papamichos et al. (eds.), Bifurcation and Degradation of Geomaterials with Engineering Applications, Springer Series in Geomechanics and Geoengineering, DOI 10.1007/978-3-319-56397-8_3

In the original version of the book, incorrect author name "Xindong Wu" has to be corrected to read as "Xingdong Wu" in Chapter 3. The erratum chapter and the book have been updated with the change. 